УДК 517.53

\title{
On Some Approach for Finding the Resultant of Two Entire Functions
}

\author{
Alexander M. Kytmanov* \\ Evgeniya K. Myshkina ${ }^{\dagger}$ \\ Institute of Mathematics and Computer Science \\ Siberian Federal University \\ Svobodny, 79, Krasnoyarsk, 660041 \\ Russia
}

Received 12.02.2019, received in revised form 27.03.2019, accepted 06.05.2019

One approach for finding the resultant of two entire functions is discussed in the article. It is based on Newton's recurrent formulas.

Keywords: resultant, entire function, Newton's formulas.

DOI: 10.17516/1997-1397-2019-12-4-434-438.

Let us consider classic resultant $R(f, g)$ for given polynomials $f$ and $g$. It can be defined in various ways:

a) using the Sylvester determinant (see, for example, [1-3]);

b) using the formula for the product $R(f, g)=\prod_{\{x: f(x)=0\}} g(x)$ (see, for example, [1-3]);

c) using the Bezout-Cayley method (see, for example, [4]).

See also monograph [5].

In our approach, we take the formula of the product as the main definition.

Let us consider the Sylvester determinant for

$$
\left\{\begin{array}{l}
f(z)=a_{0}+a_{1} z+a_{2} z^{2}+\ldots+a_{n} z^{n} \\
g(z)=b_{0}+b_{1} z+b_{2} z^{2}+\ldots+b_{m} z^{m} .
\end{array}\right.
$$

Let us define

If $a_{n} \neq 0$ then

$$
D_{n, m}=\left|\begin{array}{ccccc}
a_{0} & a_{1} & a_{2} & \ldots & 0 \\
0 & a_{0} & a_{1} & \ldots & 0 \\
\ldots & \ldots & \ldots & \ldots & \ldots \\
0 & \ldots & a_{0} & \ldots & a_{n} \\
b_{0} & b_{1} & b_{2} & \ldots & 0 \\
0 & b_{0} & b_{1} & \ldots & 0 \\
\ldots & \ldots & \ldots & \ldots & \ldots \\
0 & \ldots & b_{0} & \ldots & b_{m}
\end{array}\right|
$$

$$
D_{n, m}=a_{n}^{m} \prod_{\{x: f(x)=0\}} g(x),
$$

that is, it coincides up to a constant the multiplier with the resultant.

Let us consider the following sums of powers of the values of $g$ at the roots of $f$

$$
S_{k}=\sum_{\{x: f(x)=0\}} g^{k}(x), \quad k=1,2, \ldots .
$$

*AKytmanov@sfu-kras.ru

$\dagger$ elfifenok@mail.ru

(C) Siberian Federal University. All rights reserved 
It is known that (see [6])

$$
R_{f, g}=\frac{1}{n !}\left|\begin{array}{ccccc}
S_{1} & 1 & 0 & \ldots & 0 \\
S_{2} & S_{1} & 2 & \ldots & 0 \\
\ldots & \ldots & \ldots & \ldots & \ldots \\
S_{n} & S_{n-1} & S_{n-2} & \ldots & S_{1}
\end{array}\right|
$$

A natural generalization of polynomials are entire functions. A number of results are known that extend the definition of resultant in the case of entire functions with a finite number of zeros and with an infinite number of zeros (see [6,7]). One of the results is the following [6]. Let $g(z)$ be an entire function of the form

$$
g(z)=b_{0}+b_{1} z+b_{2} z^{2}+\ldots+b_{m} z^{m}+\ldots .
$$

then

$$
R(f, g)=\lim _{m \rightarrow \infty} a_{n}^{-m} D_{n, m} .
$$

Therefore, to find the resultant $R(f, g)$ it is necessary to calculate determinants of order $m+n$, and then to find their limit. It is certainly quite difficult. Here we propose another approach for finding the resultant.

Let us denote the roots of the polynomial $f(z)$ by $z_{1}, z_{2}, \ldots z_{n}$. Multiple roots are taken into account. Then

$$
\prod_{i=1}^{n} g\left(z_{i}\right)=g\left(z_{1}\right) \cdot g\left(z_{2}\right) \cdots g\left(z_{n}\right)=\left(\sum_{j=0}^{m} b_{j} z_{1}\right) \cdots\left(\sum_{j=0}^{m} b_{j} z_{n}\right) .
$$

This expression is the sum of some symmetric polynomials in variables $z_{1}, z_{2}, \ldots z_{n}$. These symmetric polynomials are polynomials of elementary symmetric polynomials of polynomial $f(z)$. Thus, it is possible to find the resultant of polynomials $f(z)$ and $g(z)$ without finding the roots themselves.

Let us consider an example of application of this method to the system

$$
\left\{\begin{array}{l}
f(z)=a_{0}+a_{1} z+a_{2} z^{2} \\
g(z)=b_{0}+b_{1} z+b_{2} z^{2}+\ldots+b_{m} z^{m} .
\end{array}\right.
$$

The roots of polynomial $f(z)$ from (5) are $z_{1}$ and $z_{2}$. Then

$$
\begin{gathered}
\prod_{i=1}^{2} g\left(z_{i}\right)=g\left(z_{1}\right) \cdot g\left(z_{2}\right)= \\
=b_{0}^{2}+b_{1}^{2} z_{1} z_{2}+b_{2}^{2} z_{1}^{2} z_{2}^{2}+\ldots+b_{n}^{2} z_{1}^{n} z_{2}^{n}+ \\
+b_{0} b_{1}\left(z_{1}+z_{2}\right)+b_{0} b_{2}\left(z_{1}^{2}+z_{2}^{2}\right)+\ldots+b_{0} b_{n}\left(z_{1}^{n}+z_{2}^{n}\right)+ \\
+b_{1} b_{2} z_{1} z_{2}\left(z_{1}+z_{2}\right)+b_{1} b_{3} z_{1} z_{2}\left(z_{1}^{2}+z_{2}^{2}\right)+\ldots+b_{1} b_{n} z_{1} z_{2}\left(z_{1}^{n}+z_{2}^{n}\right)+ \\
+b_{2} b_{3} z_{1}^{2} z_{2}^{2}\left(z_{1}+z_{2}\right)+b_{2} b_{4} z_{1}^{2} z_{2}^{2}\left(z_{1}^{2}+z_{2}^{2}\right)+\ldots+b_{2} b_{n} z_{1}^{2} z_{2}^{2}\left(z_{1}^{n}+z_{2}^{n}\right)+ \\
\ldots \ldots \ldots \\
+b_{n-1} b_{n} z_{1}^{n-1} z_{2}^{n-1}\left(z_{1}^{n}+z_{2}^{n}\right) .
\end{gathered}
$$

Hence we obtain 


$$
\begin{aligned}
\prod_{i=1}^{2} g\left(z_{i}\right)= & g\left(z_{1}\right) \cdot g\left(z_{2}\right)=\sum_{k=0}^{n} b_{k}^{2} z_{1}^{k} z_{2}^{k}+\sum_{t=0}^{n} \sum_{s=t+1}^{n} b_{t} b_{s}\left(z_{1}^{t} z_{2}^{s}+z_{1}^{s} z_{2}^{t}\right)= \\
& =\sum_{k=0}^{n} b_{k}^{2} z_{1}^{k} z_{2}^{k}+\sum_{t=0}^{n} \sum_{s=t+1}^{n} b_{t} b_{s} z_{1}^{t} z_{2}^{t}\left(z_{1}^{s-t}+z_{2}^{s-t}\right) .
\end{aligned}
$$

To simplify the resulting expression we use Vieta's formulas

$$
\left\{\begin{array}{l}
e_{1}=z_{1}+z_{2}=-a_{1} \\
e_{2}=z_{1} \cdot z_{2}=a_{0}
\end{array}\right.
$$

where $e_{1}, e_{2}$ are elementary symmetric polynomials of polynomial $f(z)$.

Now one needs to calculate the sums in brackets in formula (6). To do this we introduce the following notation

$$
\widetilde{S}_{k}=\sum_{i=1}^{2} z_{i}^{k}
$$

i.e.,

$$
\begin{aligned}
& \widetilde{S}_{1}=z_{1}+z_{2}, \\
& \widetilde{S}_{2}=z_{1}^{2}+z_{2}^{2}, \ldots \\
& \widetilde{S}_{k}=z_{1}^{k}+z_{2}^{k} .
\end{aligned}
$$

Expressions $\widetilde{S}_{k}$ are sums of powers of the roots of polynomial $f(z)$.

Let us consider the famous Newton-Girard formula

$$
\widetilde{S}_{k}=\sum_{r_{1}+2 r_{2}+\ldots+k r_{k}=k, r_{1}, r_{2}, \ldots, r_{k} \geqslant 0}(-1)^{k} \frac{k\left(r_{1}+\ldots+r_{k}-1\right) !}{r_{1} ! \cdot \ldots \cdot r_{k} !} \prod_{i=1}^{k}\left(-e_{i}\right)^{r_{i}} .
$$

In our case there are only two elementary symmetric polynomials. Therefore

$$
\widetilde{S}_{2 j}=\sum_{t=0}^{j}(-1)^{j+t} \frac{2 j \cdot(j+t-1) !}{(2 t) ! \cdot(j-t) !}\left(e_{1}\right)^{2 t} \cdot\left(e_{2}\right)^{j-t}
$$

if $j$ is even, and

$$
\widetilde{S}_{2 j+1}=\sum_{t=0}^{j}(-1)^{j+t} \frac{(2 j+1) \cdot(j+t) !}{(2 t+1) ! \cdot(j-t) !}\left(e_{1}\right)^{2 t+1} \cdot\left(e_{2}\right)^{j-t}
$$

if $j$ is odd

For example,

$$
\begin{gathered}
\widetilde{S}_{2}=e_{1}^{2}-2 e_{2}=a_{1}^{2}-2 a_{0}, \\
\widetilde{S}_{3}=e_{1}^{3}-3 e_{1} e_{2}=-a_{1}^{3}+3 a_{0} a_{1} .
\end{gathered}
$$

As a result, we obtain

$$
\prod_{i=1}^{2} g\left(z_{i}\right)=\sum_{k=0}^{n} b_{k}^{2} z_{1}^{k} z_{2}^{k}+\sum_{t=0}^{n} \sum_{s=t+1}^{n} b_{t} b_{s} z_{1}^{t} z_{2}^{t}\left(z_{1}^{s-t}+z_{2}^{s-t}\right)=\sum_{k=0}^{n} b_{k}^{2} a_{0}^{k}+\sum_{t=0}^{n} \sum_{s=t+1}^{n} b_{t} b_{s} a_{0}^{t} \widetilde{S}_{s} .
$$

Now, if $g(z)$ is an entire function then formula (9) takes the form 


$$
\prod_{i=1}^{2} g\left(z_{i}\right)=\sum_{k=0}^{\infty} b_{k}^{2} a_{0}^{k}+\sum_{t=0}^{\infty} \sum_{s=t+1}^{\infty} b_{t} b_{s} a_{0}^{t} \widetilde{S}_{s} .
$$

Example 1. Let us consider the system of equations

$$
\left\{\begin{array}{l}
f(z)=z^{2}-a^{2} \\
g(z)=b_{0}+b_{1} z+b_{2} z^{2}+\ldots+b_{n} z^{n} .
\end{array}\right.
$$

The roots of the first equation of the system are $\pm a$. Using formula (7), we have

$$
\prod_{i=1}^{2} g\left(z_{i}\right)=\sum_{k=0}^{n} b_{k}^{2} z_{1}^{k} z_{2}^{k}+\sum_{t=0}^{n} \sum_{s=t+1}^{n} b_{t} b_{s} z_{1}^{t} z_{2}^{t}\left(z_{1}^{s-t}+z_{2}^{s-t}\right) .
$$

Then

$$
\left\{\begin{array}{l}
e_{2}=z_{1} \cdot z_{2}=-a^{2} \\
e_{1}=z_{1}+z_{2}=0
\end{array}\right.
$$

and

$$
\widetilde{S}_{s-t}=z_{1}^{s-t}+z_{2}^{s-t}=\left\{\begin{array}{l}
2 a^{s-t}, s-t \text { even } \\
0, s-t \text { odd }
\end{array}\right.
$$

Thus

$$
\begin{gathered}
\prod_{i=1}^{2} g\left(z_{i}\right)=\sum_{k=0}^{n}(-1)^{k} b_{k}^{2} a^{2 k}+\sum_{t=0}^{n} \sum_{s=t+1}^{n}(-1)^{t} b_{t} b_{s} a^{2 t} \widetilde{S}_{s-t}= \\
=\sum_{k=0}^{n}(-1)^{k} b_{k}^{2} a^{2 k}+2 \sum_{t=0}^{n} \sum_{s=t+2}^{n}(-1)^{t} b_{t} b_{s} a^{s-t},
\end{gathered}
$$

provided that $s-t$ is an even number. Let us introduce the following designation $s-t=2 j$. Then

$$
\prod_{i=1}^{2} g\left(z_{i}\right)=\sum_{k=0}^{n}(-1)^{k} b_{k}^{2} a^{2 k}+2 \sum_{t=0}^{n} \sum_{j=1}^{[(n-t) / 2]}(-1)^{t} b_{t} b_{t+2 j} a^{2 t+2 j} .
$$

Example 2. Let us consider the system of equations

$$
\left\{\begin{array}{l}
f(z)=z^{2}-a^{2} \\
g(z)=e^{b z}=\sum_{n=1}^{\infty} \frac{(b z)^{n}}{n !}=1+b z+\frac{(b z)^{2}}{2 !}+\ldots+\frac{(b z)^{n}}{n !}+\ldots
\end{array}\right.
$$

Using formula (4), we obtain

$$
\prod_{i=1}^{2} g\left(z_{i}\right)=\sum_{k=0}^{\infty}(-1)^{k} \frac{(a b)^{2 k}}{(k !)^{2}}+2 \sum_{t=0}^{\infty} \sum_{j=1}^{\infty} \frac{(-1)^{t}(a b)^{2 t+2 j}}{t !(t+2 j) !}=1 .
$$

The first sum is the Bessel function of the first kind $J_{0}(2 a b)$ (see, for example, [8]).

The work was supported by RFBR, grant 18-51-41011 Uzb.t and grant 18-31-00019. 


\section{References}

[1] B.L.Van der Waerden, Modern Algebra, Frederick Ungar, New York, 1950.

[2] A.Kurosh, Higher Algebra, Mir, 1972.

[3] S.Lang, Algebra, Graduate Texts in Mathematics, Springer, 2002.

[4] M.G.Krein, M.A.Naimark, The method of symmetric and Hermitian forms in the theory of the roots of algebraic equation, Linear and Multilinear Algebra, 10(1981), 265-308.

[5] V.Bykov, A.Kytmanov, M.Lazman, M.Passare, Elimination Methods in Polynomial Computer Algebra, Kluwer Acad. Publishers, Dordreht, 1998.

[6] A.M.Kytmanov, Ya.M.Naprienko, One approach to finding the resultant of two entire functions, Complex variables and elliptic equations, 62(2017), no. 2, 269-286.

[7] N.G.Chebotarev, Collected Works, Vol. 2, Moscow-Leningrad, AN USSR, 1949 (in Russian).

[8] F.Bowman, Introduction to Bessel Functions, Dover, New York, 1958.

\section{О некотором подходе к нахождению результанта двух целых функций}

\section{Александр М. Кытманов}

Евгения К. Мышкина

Институт математики и фундаментальной информатики Сибирский федеральный университет Свободный, 79, Красноярск, 660041

Россия

В статье обсуждается один подход к нахождению результанта двух иелых функций, основанный на рекуррентных формулах Нъютона.

Ключевые слова: результирующие, целье функиии, формуль Ньютона. 